\title{
DOPPLER FLOW VELOCITY WAVEFORMS IN THE FETAL CARDIAC OUTFLOW TRACT: REPRODUCIBILITY OF WAVEFORM RECORDING AND ANALYSIS
}

\author{
Irene A. L. Groenenberg, ${ }^{\dagger}$ WiM C. J. Hop ${ }^{\ddagger}$ and JuriY W. WLAdimirofF ${ }^{\dagger}$ \\ ${ }^{\dagger}$ Department of Obstetrics and Gynecology and ${ }^{\ddagger}$ Department of Epidemiology and Biostatistics. \\ Erasmus University Rotterdam, Rotterdam, The Netherlands
}

(Received I October 1990: in final form 31 January 1991)

\begin{abstract}
Reproducibility of flow velocity waveform recording and analysis was studied at fetal cardiac level (ductus arteriosus, pulmonary artery and ascending aorta) in 42 normal pregnancies. The flow velocity parameters studied were the peak systolic velocity (PSV), acceleration time (ACT), acceleration velocity (ACV), average velocity (AV) and flow velocity integral (FVI). In each patient, two consecutive measurements were performed (time delay $15 \mathrm{~min}$ ) and of each measurement two hardcopies were analysed. A high reproducibility was achieved for the PSV, AV and FVI in all vessels studied; the coefficients of variation between readings of hardcopies were $\leq 3 \%$, and the coefficients of variation between tests within patients were $\leq 7 \%$. A moderate reproducibility was achieved for the ACT in the ascending aorta and pulmonary artery; the variation between tests was large for the ductus arteriosus. The reproducibility of the $A C V$ was poor.
\end{abstract}

Key Words: Fetal cardiac outflow tract, Doppler ultrasonography, Reproducibility.

\section{INTRODUCTION}

An increasing number of reports has appeared on flow velocity waveform recording in the fetal cardiac outflow tract (Allan et al. 1987; De Smedt et al. 1987; Hata et al. 1987; Huhta et al. 1987; Kenny et al. 1986; Reed et al. 1987). Flow velocity waveforms are influenced by various factors such as preload, afterload (including arterial pressure and vascular resistance), heart rate and the intrinsic contractile properties of the left and right ventricle. The human fetal model does not allow differentiation between these factors (Groenenberg et al. 1989). However, little information is available on the reproducibility of waveform recording and analysis at this level of the fetal circulation. There is only one report available on the interobserver variability of vessel diameter and flow velocity integral measurements in the fetal cardiac outflow tract (Kenny et al. 1986). A good agreement between two observers was found for the analyses of each re-

Address correspondence to: J. W. Wladimiroff, M.D., Ph.D. Professor of Obstetrics and Gynecology, Academic Hospital Rotterdam-Dijkzigt, Erasmus University Rotterdam, Dr. Molewaterplein 40,3015 GD Rotterdam. The Netherlands.

This study was supported by the Dutch Foundation for Medical Research MEDIGON (Grant No. 900-516-105) cording. The variation between analyses within observers, however, was not assessed. The same applied to within-patients variation between recordings. The present study was designed to assess (a) the variations of blood flow velocity waveform recordings between repeated tests within patients, and (b) the reproducibility of the analysis of the recordings. Blood flow velocity recordings were obtained from the fetal ascending aorta, pulmonary artery and ductus arteriosus in the normal second half of pregnancy.

\section{MATERIALS AND METHODS}

A total of 42 patients consented to participate in the study. Gestational age varied between 22 and 34 weeks (mean 29 weeks). All pregnancies were uncomplicated, with the fetal abdominal circumference between the 5th and 95th percentile of the reference chart (Campbell and Wilkin 1975) and birthweight between the 5th and 95th percentile (Kloosterman 1970). Pregnancy duration was determined from the last menstrual period and confirmed by ultrasonic measurements of the biparietal diameter at 14-22 weeks.

A combined mechanical sector and pulsed/continuous Doppler system (Diasonics CV 400, Diason- 
ics Inc., Mulpikas, CA) with a carrier frequency of 3.5 (real time) and $3.0 \mathrm{MHz}$ (Doppler) was used. The sector scanner operates at power outputs of less than 100 $\mathrm{mW} / \mathrm{cm}^{2}$ spatial peak-temporal average in both imaging and Doppler modes according to the manufacturer's specifications. The frequency analysis device of the Diasonics CV $\mathbf{4 0 0}$ is a fast Fourier transformer spectrum analyzer. The data collection time (fast Fourier transit time, FFT) of this spectrum analyzer is $20 \mathrm{~ms}$.

All Doppler studies were performed with the patient in the semirecumbent position and during periods of fetal apnea, because high-amplitude fetal breathing movements modulate blood flow velocity waveforms (Marsal et al. 1984). Measurements were made irrespective of fetal activity or heart rate accelerations. All measurements were performed by one investigator (I. A. L. G.). Doppler measurements were performed in the ascending aorta, pulmonary artery and ductus arteriosus. Two-dimensional real-time imaging was necessary to position correctly the Doppler sample volume in the vessel of interest. The correct position of the sample volume was verified after each Doppler measurement. Flow velocity waveforms from the ascending aorta, pulmonary artery and ductus arteriosus were obtained from the fivechamber view (Kenny et al. 1986), the conventional short axis view (Kenny et al. 1986) and the ductal plane (Huhta et al. 1987), respectively. Pulsed Doppler was used for flow velocity waveform recording in the ascending aorta and pulmonary artery. A sample volume length of $0.3 \mathrm{~cm}$ was used. The sample volume was placed immediately distal to the semilunar valves to obtain flow velocity waveforms with a narrow Doppler spectrum envelope reflecting the flat velocity profile existing in the great vessels. Continuous wave Doppler was used for flow velocity waveform recording in the ductus arteriosus. The high velocities registered in this vessel require a continuous Doppler system, as this is not subject to frequency aliasing. Recordings were only accepted if the angle between the interrogating Doppler beam and the direction of the blood flow was estimated at 15 degrees or less. No angle correction was used. For all vessels studied, peak systolic velocity (PSV in $\mathrm{cm} / \mathrm{s}$ ), acceleration time ( $\mathrm{ACT}$ in $\mathrm{ms}$ ), acceleration velocity ( $\mathrm{ACV}$ in $\mathrm{m} / \mathrm{s}^{2}$ ), average velocity ( $\mathrm{AV}$ in $\mathrm{cm} / \mathrm{s}$ ) and flow velocity integral (FVI in $\mathrm{cm}$ ) were determined. An average of four consecutive flow velocity waveforms of similar appearance and high signal-noise ratio was used to establish each value. All Doppler waveforms were recorded on videotape. From this tape, hardcopies were made, consisting of four to five flow velocity wave- forms (depending on fetal heartrate), that is, two seconds of recording. A flow velocity waveform with a period time of $400 \mathrm{~ms}$ will therefore measure 26.5 $\mathrm{mm}$ on the $X$ axis of the hardcopy. For measurements performed in the ascending aorta and pulmonary artery, a velocity of $50 \mathrm{~cm} / \mathrm{s}$ corresponded with $5 \mathrm{~cm}$ on the $Y$ axis of the hardcopy, whereas for the ductus arteriosus this corresponded with $2.5 \mathrm{~cm}$ on the $Y$ axis of the hardcopy. An IBM-compatible microcomputer (Olivetti M24, Olivetti BV, Leiden, The Netherlands) linked to a graphics tablet (MM 1201, Summagraphics) was used for analysis of the Doppler recordings on the hardcopies. Resolution of the graphics tablet was $.05 \mathrm{~mm}$. The analysing program uses 400 datapoints to describe the four waveforms on one hardcopy. Resolution of the analysing programme was $.325 \mathrm{~mm}$ for the $X$ axis and $.5 \mathrm{~mm}$ for the $Y$ axis of the hardcopy. Flow velocity waveform analysis consisted of tracing the outer border of the densest part of the Doppler spectrum envelope of each waveform with a cursor and defining the onset, maximum and end of the waveform. PSV $(\mathrm{cm} / \mathrm{s})$ was defined by the top of the densest part of the Doppler spectrum envelope. ACT (ms) was defined as the time interval between the onset of the waveform and the peak systolic velocity. The datapoints between $10 \%$ and $90 \%$ of the ascending limb of the curve were used to construct a regression line and thus estimate the ACV $\left(\mathrm{m} / \mathrm{s}^{2}\right)$. AV $(\mathrm{cm} /$ $\mathrm{s})$ was calculated by dividing the sum of velocities over one period time by the number of datapoints. The FVI $(\mathrm{cm})$ was obtained by multiplying the average velocity with the period time.

\section{Reproducibility study}

In each patient, Doppler recordings were performed twice in all three cardiac vessels, at times $t_{1}$ and $t_{2}$. The time delay between the two measurements was approximately 15 minutes. Of each recording, two hardcopies were made. These hardcopies did not reveal the identity or gestational age of the patient nor the date or time of recording. After collecting the hardcopies, they were shuffled in a random order and analysed. Both Doppler recording and waveform analysis was performed by the same investigator (I. A. L. G.). Statistical analysis consisted of Nested Analysis of Variance to separate the total variation in components due to differences between patients, differences between repeated tests within patients and differences between analyses of hardcopies. Linear relations between variables were assessed using Pearson's correlations. Other methods used are given in the text. Statistical significance was set at $P=.05$ (two-sided). 


\section{RESULTS}

Unacceptable flow velocity waveforms were obtained in one patient (34 weeks of gestation) due to severe obesity, leaving data from 41 patients for further analysis. Acceptable paired recordings of the first Doppler measurement at $t_{1}$ and second Doppler measurement at $t_{2}$ were obtained from the ductus arteriosus in 26 patients, from the pulmonary artery in 29 patients and from the ascending aorta in 31 patients. If one of the two Doppler recordings was unsuccessful in a particular vessel, the entire recording was omitted from further analysis, since it did not contribute to the analysis of variation between tests. The success rate in obtaining paired recordings in the ductus arteriosus correlated positively with the success rate in collecting paired recordings in the pulmonary artery, and vice versa (Fisher exact test: $P<.05$ ). The success rate in obtaining acceptable paired recordings at $t_{1}$ and $t_{2}$ from the ductus arteriosus, pulmonary artery or ascending aorta did not correlate with gestational age. Table 1 gives standard deviations corresponding to the three sources of variation for each vessel. The best results, both for the variation in hardcopy analyses as well as the variation between repeated tests, were obtained for the peak systolic velocity. Figure 1 shows test results for this parameter for individual patients.

For all Doppler parameters, the standard deviations corresponding to the two consecutive tests did not significantly correlate with gestational age. The difference in fetal heart rate between the two consecutive tests was less than $11 \mathrm{bpm}$ in $90 \%$ of tests (range $0-19$, median $4 \mathrm{bpm}$ ). In reanalysing the data, confining the analysis to tests in which the fetal heart rate differed $\leq 10 \mathrm{bpm}$, the resulting components of variance did not appreciably differ from those given in Table 1 .

\section{DISCUSSION}

The present results show that analyses from hardcopies can be performed with a high reproducibility for the parameters PSV, AV and FVI. Also, the variation between repeated tests at an interval of 15 minutes is small for these parameters. The reproducibility of hardcopy analyses for ACT appears smaller as compared with PSV, AV and FVI, but still seems acceptable. The variation between tests, however, is considerable. This is particularly so for the flow velocity waveform recordings from the ductus arteriosus. A poor reproducibility was established for the ACV from the analyses of hardcopies, while there were also considerable differences between repeated tests for the ascending aorta and ductus arteriosus. Variation due to the tracing hardcopies is unacceptably large for using this cardiac parameter in describing cardiac dynamics under any circumstances. An acceptable explanation for this poor reproducibility is found in the steepness of the ascending limb of the flow velocity waveform. The ascending limb is too steep to be traced accurately by hand. Another explanation is found in the relatively long FFT of $20 \mathrm{~ms}$. The fast Fourier transformer spectrum analyzer tests for a set of frequencies and displays the intensities of each of the frequencies present as a function of time in the form of a spectral waveform (Beach 1987). For each spectral estimate, a short portion of the signal is used. The longer the data collection time (FFT) used for

Table 1. Standard deviations (coefficients of variation between parentheses) derived from Analysis of Variance.

\begin{tabular}{|c|c|c|c|c|c|}
\hline & $\mathrm{PSV}(\mathrm{cm} / \mathrm{s})$ & $\mathrm{ACT}(\mathrm{ms})$ & $\operatorname{ACV}(\mathrm{m} / \mathrm{s})$ & $\mathrm{AV}(\mathrm{cm} / \mathrm{s})$ & FVI $(\mathrm{cm})$ \\
\hline \multicolumn{6}{|c|}{ DA } \\
\hline $\begin{array}{l}\sigma_{p} \\
\sigma_{t} \\
\sigma_{c}\end{array}$ & $\begin{array}{c}16.7(18 \%) \\
3.2(3 \%) \\
0.9(1 \%)\end{array}$ & $\begin{array}{c}11.2(20 \%) \\
9.7(17 \%) \\
2.9(5 \%)\end{array}$ & $\begin{array}{l}5.6(27 \%) \\
2.9(14 \%) \\
3.9(19 \%)\end{array}$ & $\begin{array}{l}5.5(16 \%) \\
2.0(6 \%) \\
0.6(2 \%)\end{array}$ & $\begin{array}{l}2.9(20 \%) \\
1.0(7 \%) \\
0.3(2 \%)\end{array}$ \\
\hline $\begin{array}{l}\sigma_{p} \\
\sigma_{t} \\
\sigma_{c}\end{array}$ & $\begin{array}{l}9.4(17 \%) \\
1.2(2 \%) \\
0.6(1 \%)\end{array}$ & $\begin{array}{l}7.8(15 \%) \\
5.1(10 \%) \\
4.3(8 \%)\end{array}$ & $\begin{array}{l}2.7(20 \%) \\
0.0(0 \%) \\
4.9(36 \%)\end{array}$ & $\begin{array}{l}3.1(18 \%) \\
0.8(5 \%) \\
0.4(2 \%)\end{array}$ & $\begin{array}{l}1.4(19 \%) \\
0.3(4 \%) \\
0.2(3 \%)\end{array}$ \\
\hline $\begin{array}{l}\sigma_{p} \\
\sigma_{t} \\
\sigma_{c}\end{array}$ & $\begin{array}{l}8.8(12 \%) \\
1.8(2 \%) \\
0.6(1 \%)\end{array}$ & $\begin{array}{l}5.2(10 \%) \\
5.3(11 \%) \\
4.2(8 \%)\end{array}$ & $\begin{array}{l}3.5(17 \%) \\
2.8(14 \%) \\
6.6(32 \%)\end{array}$ & $\begin{array}{l}2.8(14 \%) \\
0.9(4 \%) \\
0.6(3 \%)\end{array}$ & $\begin{array}{l}1.2(14 \%) \\
0.4(5 \%) \\
0.2(2 \%)\end{array}$ \\
\hline
\end{tabular}

$\mathrm{DA}=$ ductus arteriosus; $\mathrm{PA}=$ pulmonary artery $\mathrm{AO}=$ ascending aorta; $\mathrm{PSV}=$ peak systolic velocity; $\mathrm{ACT}=$ acceleration time; $\mathrm{ACV}=$ acceleration velocity; $\mathrm{AV}=$ average velocity; $\mathrm{FVI}=$ flow velocity integral. $\sigma_{p}$ corresponds to differences between patients, $\sigma_{t}$ to differences between repeated tests (time delay $15 \mathrm{~min}$ ) within patients and $\sigma_{c}$ to differences between readings of hardcopies. 

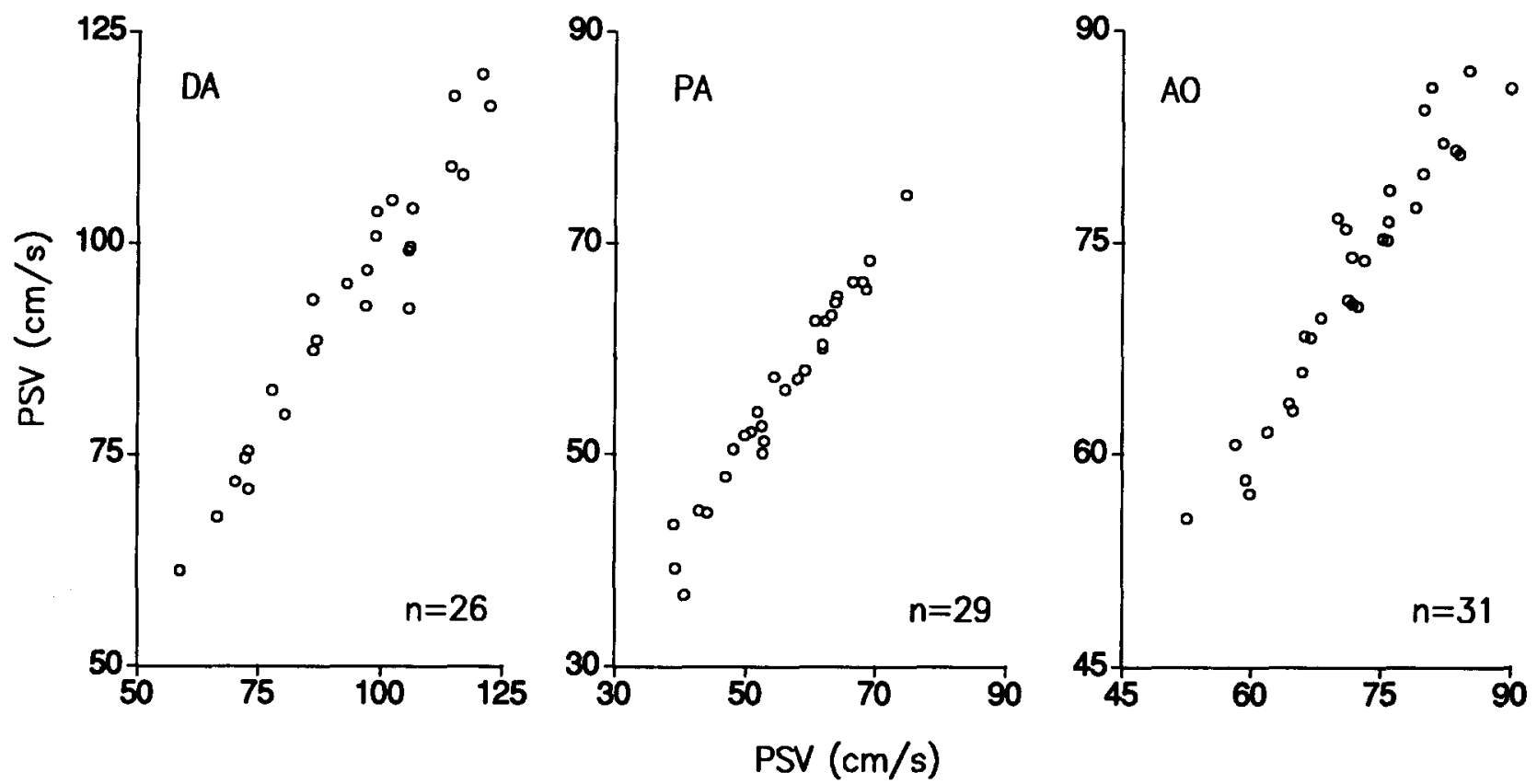

Fig. 1. Peak systolic velocity (PSV) at second test (vertical) versus first test (horizontal) for DA, PA and AO. Plotted data are the means of two hardcopy readings. DA = ductus arteriosus; $\mathrm{PA}=$ pulmonary artery; $\mathrm{AO}=$ ascending aorta.

spectral estimate, the larger the inaccuracies in estimation of velocity changes. The relatively long data collection time (FFT) of $20 \mathrm{~ms}$ limits accurate estimation of high-rate velocity changes by the spectrum analyzer.

The moderate reproducibility for the ACT can also be partly explained by the fast Fourier transit time (FFT): Suspicion arises as to whether the FFT enables accurate determination of the ACT because the FFT of $20 \mathrm{~ms}$ is large compared to the mean ACT of about 40 ms. Moreover, several reports have appeared on the effect of sample volume positioning regarding the ACT (Panadis et al. 1986, Shaffer et al. 1990).

In the present study, the sample volume was always placed immediately distal to the valves. No considerable differences in fetal heartrate were observed between the two consecutive tests. The large betweentests variation which was observed for some parameters is therefore not largely affected by variations in heart rate. Reanalysis of data, leaving out those with a difference of more than $10 \mathrm{bpm}$ between the two tests, confirmed this finding. The standard deviation corresponding to the two consecutive measurements does not significantly increase or decrease with gestational age, indicating gestational age independency in our reproducibility study.

The positive correlation between the ductus arteriosus and the pulmonary artery in successfully ob- taining an acceptable Doppler measurement is not surprising. Due to the close anatomical relationship between the two vessels, only a minor change in scanning plane is necessary to obtain a flow velocity waveform from the other vessel.

\section{CONCLUSION}

At the level of the fetal cardiac outflow tract, a high reproducibility in determining PSV, AV and FVI can be achieved, indicating that these cardiac parameters can be used for assessing cardiac function. Whereas a moderate reproducibility was achieved for the ACT in the ascending aorta and the pulmonary artery, the reproducibility in the ACT of the ductus arteriosus and the $\mathrm{ACV}$ in all vessels studied was poor.

\section{REFERENCES}

Allan, L. D.; Chita, S. K.; Al-Ghazali, W.; Crawford, D. C.; Tynan, M. Doppler echocardiographic evaluation of the normal human fetal heart. Br. Heart J. 57:528-533; 1987.

Beach, K. W. Research lessons for maternal-fetal studies from Doppler studies of vascular disease. In: Maulik, D.; McNellis, D., eds. Reproductive and perinatal medicine (VIII). New York: Perinatology Press; 1987:225-246.

Campbell, S.; Wilkin, D. Ultrasonic measurements of the fetal abdominal circumference in estimation of fetal weight. $\mathrm{Br}$. J. Obstet. Gynaecol. 82:689-696; 1975.

De Smedt, M. C. H.; Visser, G. H. A.; Meijboom, E. J. Fetal cardiac output estimated by Doppler echocardiography during midand late gestation. Am. J. Cardiol. 60:338-342; 1987.

Groenenberg, I. A. L.; Wladimiroff, J. W.; Hop, W. C. J. Fetal 
cardiac and peripheral arterial flow velocity waveforms in intrauterine growth retardation. Circulation 80:1711-1717; 1989.

Hata, T.; Showa, A.; Hata, K.; Kitao, M. Intracardiac blood flow velocity waveforms in normal fetuses in utero. Am. J. Cardiol. 59:464-468; 1987.

Huhta, J. C.; Moise, K. L.; Fisher, D. J.; Sharif, D. S.; Wasserstrum, $\mathrm{N}$.; Martin, C. Detection and quantitation of constriction of the fetal ductus arteriosus by Doppler echocardiography. Circulation 75:406-412; 1987.

Kenny, J. F.; Plappert, T.; Doubilet, P.; Saltzman, D. H.; Cartier, M.; Zollars, L.; Leatherman, G. F.; St. John Sutton, M. G. Changes in intracardiac blood flow velocities and right and left stroke volumes with gestational age in the normal human fetus: A prospective Doppler echocardiographic study. Circulation $74: 1208-1216 ; 1986$.
Kloosterman, G. J. On intrauterine growth. Int. J. Gyneacol. Obstet. 8:895-913; 1970.

Marsal, K.; Eik-Nes, S. H.; Lindblad, A.; Lingman, G. Blood flow in the fetal descending aorta: Intrinsic factors affecting fetal blood flow, i.e., fetal breathing movements and cardiac arrhythmia. Ultrasound Med. Biol. 10:339-348; 1984.

Panadis, I. P.; Ross, J.; Mintz, G. S. Effect of sampling site on assessment of pulmonary artery blood flow by Doppler echocardiography. Am. J. Cardiol. 58:1145-1147; 1986.

Reed, K. L.; Anderson, C. F.; Shenker, L. Fetal pulmonary artery and aorta: Two-dimensional Doppler echocardiography. Obstet. Gynecol. 69:175-178; 1987.

Shaffer, E. M.; Snider, R. A.; Serwer, G. A.; Peters, J.; Reynolds, P. A. Effect of sampling site on Doppler derived right ventricular systolic time intervals. Am. J. Cardiol. 65:950-952; 1990. 